\title{
3D Printed UWB Microwave Bodyscope for Biomedical Measurements
}

\author{
S. Rashid, L. Jofre, Fellow, IEEE, A. Garrido, G. Gonzalez, Y. Ding, Senior Member, IEEE, A. Aguasca, J. \\ O'Callaghan, Senior, IEEE and J.Romeu, Fellow, IEEE
}

\begin{abstract}
In this letter a 3D printed compact UWB extended gap ridge horn (EGRH) antenna designed to be used for biological measurements of the human body is described. The operational frequency covers the microwave band of interest from 0.5 to $3.0 \mathrm{GHz}$ (for a $S_{11}$ under $-7 \mathrm{~dB}$ ). The 3D printed EGRH antenna is dielectrically matched to the permittivity of the human body and because of its compactness it can be visualized as a general purpose microwave probe among the RF biomedical community. The probe has proven its capability as a pass-through propagation sensor for different parts of the human body and as a sensor detecting a $1 \mathrm{~cm}$ diameter object placed inside an artificial head phantom.
\end{abstract}

Index Terms-3D printed, biological measurements, microwave, DRH antenna, compact antenna, UWB.

\section{Motivation}

$\mathbf{M}$ ICROWAVE technology has shown promising potential in medical diagnostic methods because of its noninvasive, non-ionizing and penetrating characteristics. Biomedical applications employing microwave technology range from monitoring vital signs [1] to sensing and imaging tissue abnormalities such as breast cancer and brain stroke's [2]-[4].

When designing antennas at microwave frequency bands for human body measurements, challenges arise in terms of dimension, bandwidth and radiation properties. Typically, unidirectional wideband antennas are required, moreover size reduction allows compact multisensor imaging devices [5]. A technique to achieve both compactness and matching is immersing antennas in a high permittivity media, thus reducing its dimensions and improving the matching between the radiator and the biological target [6]. High permittivity materials such as water [7], [8] or canola oil [9] have been proposed as immersive matching liquids. However, using liquids inside plastic or glass containers increases the overall volume of the measurement setup and difficults maintaining regular clinical sanitation of the liquid. An alternative approach is to develop applicators that are matched directly to the human body i.e. by placing them in direct contact. Good examples of such antennas include [10]-[12]. In [10] a 1.8 to $12 \mathrm{GHz}$ UWB

Manuscript received July 30, 2018. This work was supported by the Spanish "Comision Interministerial de Ciencia y Tecnologia" (CICYT) under projects TEC2013-47360-C3-1-P, TEC2016-78028-C3-1-P, MDM2016-0600. (Corresponding author: Lluis Jofre Roca)

S. Rashid and Y. Ding are with the College of Information Sciences and Technology and the Engineering Research Center of Digitized Textile and Fashion Technology, Ministry of Education, Donghua University, Shanghai 201620, China (e-mail: sabarashid89@yahoo.com, ysding@dhu.edu.cn)

L. Jofre, A. Garrido, G. Gonzalez, A. Aguasca, J. O'Callaghan and J. Romeu are with the School of Telecommunication Engineering, Universitat Politecnica de Catalunya, Barcelona, Spain (luis.jofre@upc.edu) microwave sensor contained into a cylindrical waveguide and loaded with epoxy is presented. For biomedical ultrawideband radar applications, a dielectrically scaled double ridged horn antenna made completely out of high dielectric constant sintered ceramic is developed in [11]. [12] reports a 3 to $10 \mathrm{GHz}$ TEM horn antenna embedded in a solid dielectric material for near-field microwave imaging based on aperture raster scanning. Usually the antennas feature acceptable performance, but they are bulky and complex to fabricate.

In this paper, we aim at developing a physically smallsized antenna with an idea that resembles to a microwave bodyscope [13] (an adaptation of the stethoscope concept normally used with sounds) and covers the frequency band of recent interest from 0.5 to $3.0 \mathrm{GHz}$. For this purpose, an UWB antenna is filled with a high permittivity material. For the antenna, a double ridged horn is selected because of its simple structure, its convenience to be filled with a liquid gel type material and the increase of the critical height of the ridge gap dimensions when filled with high dielectric materials. Thus, the antenna may be seen as an increased (extended) gap ridged horn (EGRH) geometry. As the gap height of the EGRH antenna when filled with a high permittivity material has feasible dimensions, it has been chosen 3D printing as the fabrication technique. The proposed EGRH antenna may be compared to the Microwave Sthetoscope [14], [15], a very compact design optimized for a close frequency band (0.7 to $1.5 \mathrm{GHz}$ ) mainly intended for in-vivo reflectometry for vital signals, designed to have certain focusing capability inside the human body at the price of a non-planar geometry.

This paper is organized as follows: Section II introduces the medium characterization and the antenna design procedure. Section III presents the fabrication steps. Section IV reports measurements to verify performance and test the feasibility of the microwave antenna experimentally on different parts of the human body and on an artificial head phantom. Finally, concluding remarks are given in Section V.

\section{Design Procedure}

As stated, the microwave probe is designed to come in direct contact with the human body and achieves a wide operating frequency bandwidth from 0.5 to $3.0 \mathrm{GHz}$ (for a $S_{11}$ under $-7 \mathrm{~dB})$. The relative permittivity of the filling material has been chosen to be 50 . This is a compromise to match the average permittivity of the brain and that of other biological tissues with high water content. This medium is fabricated based on the procedure described in [16]. A bio-dielectric 
material mimicking a mixture of the white and gray matter of the human brain (white-gray mixture), was manufactured by mixing different proportions of corn-flour, gelatin and distilled water. An open-ended coaxial line [17] (with operational bandwidth from 0.5 to $3.0 \mathrm{GHz}$ ) is used to experimentally obtain the complex permittivity of the developed material. The results for the complex permittivity of the white-gray mixture, $\varepsilon_{\mathrm{w}-\mathrm{g}}$ (real part of the permittivity and conductivity) are shown in Figure 1 and its good proximity to the real human values obtained from [18].

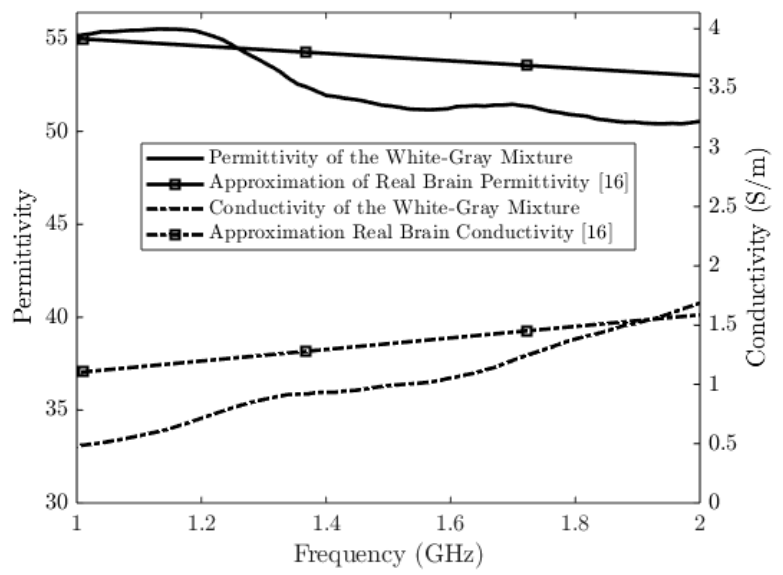

Fig. 1: Relative Permittivity and Conductivity of the fabricated White-gray mixture compared to real human values [18].

Figure 2 illustrates the geometry of the EGRH antenna whose dimensions after optimization are shown in Table I. These dimensions were later adjusted with Ansoft HFSS for the actual permittivity of the fabricated white-gray mixture for an input impedance of $50 \Omega$. The horn consists of a waveguidefeeding and a ridge-horn transition sections. In the waveguide feeding point, a coaxial enters into the waveguide from the upper ridge, and opens in the gap between the upper and lower ridges, then it enters into a lower solid coaxial line creating the equivalent of a symmetrical dipole backed by a short-circuited waveguide cavity.

TABLE I: Design dimensions of the proposed EGRH antenna

\begin{tabular}{||c|c|c||}
\hline Nomenclature & Description & Value $(\mathrm{mm})$ \\
\hline$a_{h}$ & Horn Aperture Width & 43.0 \\
$b_{h}$ & Horn Aperture Height & 30.0 \\
$l_{h}$ & Horn Length & 32.0 \\
$a_{w}$ & Waveguide Width & 20.0 \\
$b_{w}$ & Waveguide Height & 20.0 \\
$l_{w}$ & Waveguide Length & 9.0 \\
$w_{r}$ & Ridge Width & 3.3 \\
$h_{r}$ & Ridge Height & 6.3 \\
$g_{r}$ & Separation Between Ridges & 3.5 \\
\hline
\end{tabular}

Figure 3 shows the Electric Field distribution at $0.5 \mathrm{GHz}$ and $1.75 \mathrm{GHz}$ into the plane of the open-ended horn aperture and into a transversal plane perpendicular to the aperture plane for a wave propagating in the fabricated white-gray mixture.

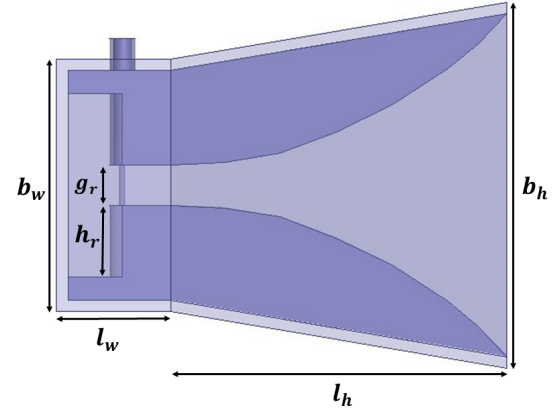

(a) Side view

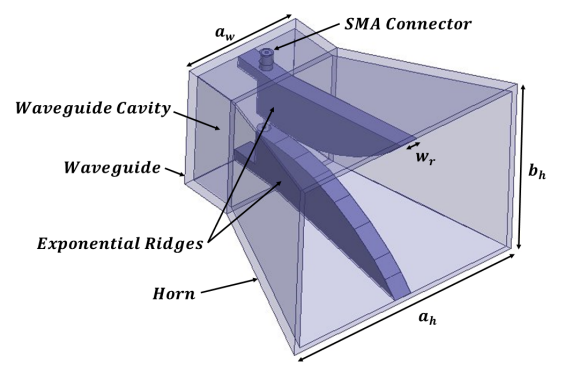

(b) $3 \mathrm{D}$ view

Fig. 2: EGRH antenna geometry

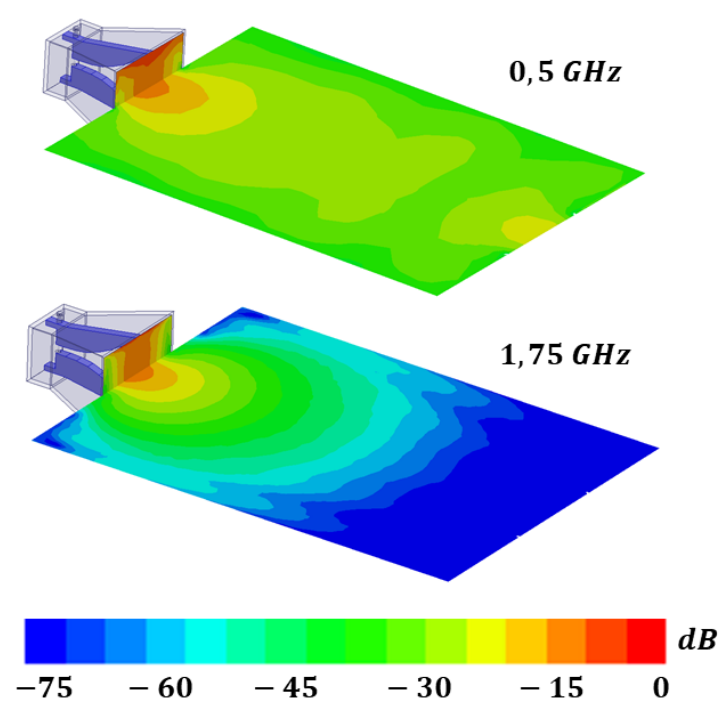

Fig. 3: Simulated E-Field distribution at two different frequencies into the plane of the open-ended horn aperture and into the transversal plane.

\section{FABRicAtion of the 3D PRINTED ANTENNA}

The process of fabrication is organized into two main steps:

1) $3 \mathrm{D}$ Printing and Metallization of the antenna following the procedure described in [19].

2) Assembling, filling and protecting: first the coaxial cable is inserted and fixed to the horn, then the antenna is filled with the white-gray dielectric matching material after applying a thin rubber coating layer. Finally, the antenna is dipped in a thick air-dry peeling rubber coating (Plastidip) providing protection against the water evaporation, and the external conditions that could produce changes 
in the permittivity of the material.

Following these fabrication steps, two antenna prototypes were successfully manufactured and tested. Images of the fabricated antennas are shown in Figure $4 \mathrm{a}$ and $4 \mathrm{~b}$.

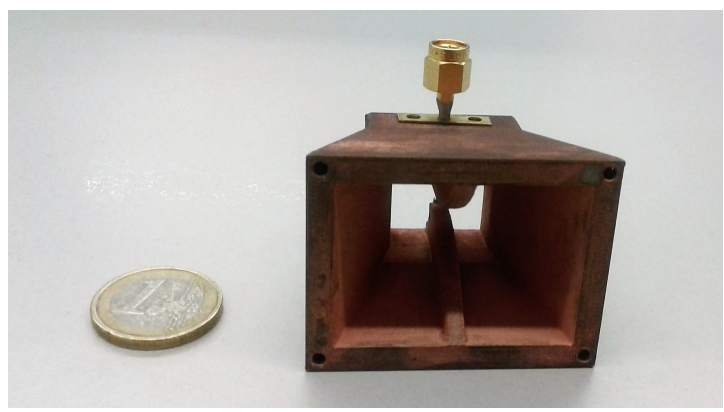

(a) Fabricated 3D printed metallized antenna prototype

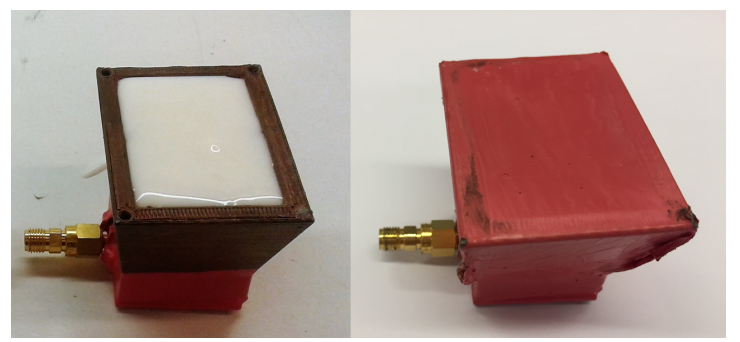

(b) Antenna filled with dielectric material and rubber coating

Fig. 4: Manufacturing process of the EGRH antenna

\section{EXPERIMENTAL RESULTS}

\section{A. Experimental setup description and calibration}

An experimental setup has been built to validate the performance of the the EGRH antenna. The setup consists on a rectangular box $\left(180.0 \times 128.0 \times 95.0 \mathrm{~mm}^{3}\right)$ with longitudinal and transversal dimensions close to those of an average human head (Figure 5). Two EGRH probe antennas may work either independently or attached into the opposite longitudinal vertical walls of the rectangular box. Both the probes and the box are filled with the fabricated white-gray mixture material. The setup is used to measure first the performance of the two probes and then as a phantom of the human brain to conduct a set of measurements such as propagation losses and reflectometric location. To perform these measurements, the EGRH antennas are connected to an Agilent E5071C Vector Network Analyzer to measure and calibrate the reflection $\left(S_{11}\right)$ and transmission $\left(S_{21}\right)$ parameters.

\section{B. Propagation losses into biological human media}

Based into the previous calibration, different propagation measurements have been conducted to explore the capabilities of the different microwave frequencies to propagate through different body parts and to extract the corresponding attenuation losses. When performing wireless measurements, the transmission coefficient $S_{21}$, the ratio between the received
$\left(P_{r}\right)$ and transmitted $\left(P_{t}\right)$ power, may be related to the propagation values as:

$$
\begin{aligned}
\left|S_{21}\right|^{2} & =\frac{P_{r}}{P_{t}}=\left(\frac{A_{e f}}{\lambda_{e} r_{T R}} e^{-\alpha_{m}(f) r_{T R}}\right)^{2}= \\
& =\left(\frac{A_{e f} f \sqrt{\varepsilon_{w-g}}}{c_{0} r_{T R}} e^{-\alpha_{m}(f) r_{T R}}\right)^{2}
\end{aligned}
$$

Where $A_{e f}$ is the effective area of the EGRH horn antenna, $r_{T R}$ is the distance between the two transmitting and receiving antennas, $\lambda_{e}$ is the wavelength inside the propagation medium, $c_{0}$ is the vacuum velocity of light, $f$ the operating frequency and $\alpha_{m}(f)$ the losses into the medium. When using mediumgain antennas, having an aperture that may be considered electrically large enough $\left(a_{h} b_{h}>\lambda^{2}\right)$, it may be accepted that $A_{e f} \cong$ constant with frequency. From Eq. 1, it may be then seen that for a given $r_{T R}$ when the frequency increases the $\left|S_{21}\right|^{2}=\frac{P_{r}}{P_{t}}$ may have a compensatory effect with the increment of losses with frequency $\left(\alpha_{m}(f)\right)$. Using the two calibrated probe antennas, four different measurements have been performed (setup box with white-gray mixture material, and a human head, neck and thorax) and the corresponding $\alpha_{m}(f)$ losses have been extracted using Eq. 1. Figure 6 represents the measured $S_{11}$ for the EGRH antenna radiating into the rectangular box (Figure 5). The difference between the simulated and the measured values, especially above $1.5 \mathrm{GHz}$ are basically due to the difference between the intended permittivity values for the white-gray mixture and the fabricated ones (as seen in Figure 1), resulting into real values just below $-7 \mathrm{~dB}$. Figure 7 depicts the experimental transmission $S 12$ (frequency upper limit fixed by the actual noise level) through three different human parts (brain, neck, thorax) measured on a real young human volunteer, where it may be seen how propagating through thick parts of the human body above $2 \mathrm{GHz}$ may imply overcoming losses above $80 \mathrm{~dB}$. Finally, Figure 8 shows the corresponding attenuation values in $\mathrm{dB} / \mathrm{cm}$. The measured values approach well those presented in the literature. As an example, the values for the brain losses, both theoretical [20] and experimental [5], published in the literature, with average losses at $1 \mathrm{GHz}$ of 1.8 to $2.0 \mathrm{~dB} / \mathrm{cm}$, match quite well (5\% error) the values depicted in Figure 8 $(2.1 \mathrm{~dB} / \mathrm{cm})$.

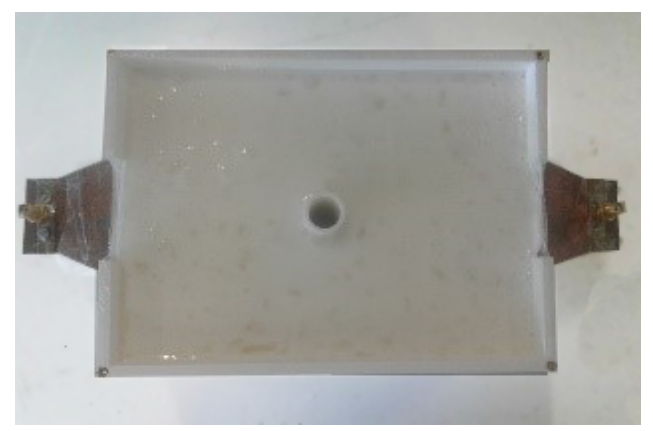

Fig. 5: Phantom $\left(180.0 \times 128.0 \times 95.0 \mathrm{~mm}^{3}\right)$ printed and filled, with the EGRH antennas. 


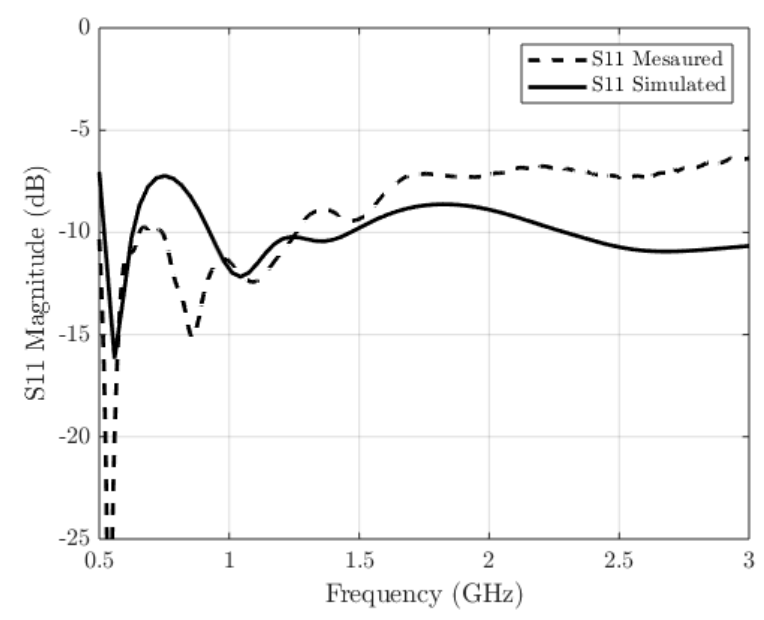

Fig. 6: Reflection Coefficient of the EGRH antenna in the Phantom Box.

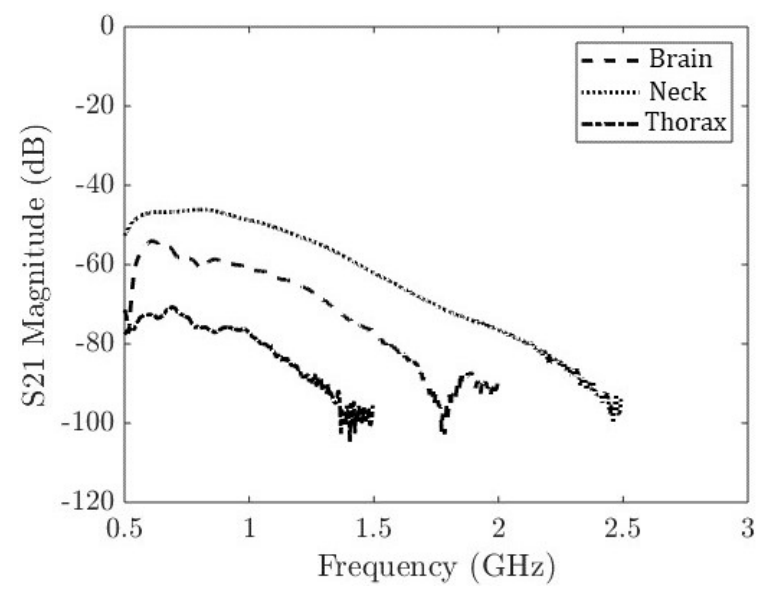

Fig. 7: Transmission Coefficient of the EGRH antenna in the Human Body.

\section{Reflectometry location into biological human media}

A second test of the potential of the probes, was to study their capability to detect a scattering object located at different depths inside the biological material. With this in mind, a metallic $1 \mathrm{~cm}$ diameter cylinder is located inside the rectangular phantom box at successive distances of 2.5, 5.0 and $10.0 \mathrm{~cm}$ from one of the probes. After calibrating the VNA, the reflection coefficient $S_{11}$ is measured in the frequency domain for each location of the metallic cylinder, and the FFT was computed and time to distance converted to determine the position of the object (Figure 9) showing a very good accuracy for the location of the scattering object. The different values of the corresponding amplitudes match with the actual losses into the medium.

\section{CONClusion}

A compact 3D printed microwave probe (compact bodyscope) for the estimation of the propagation losses and reflectometry inside a biological medium is presented. The proposed EGRH antenna attains an UWB 1:6 bandwidth, compact volume (width $\mathrm{x}$ height $\mathrm{x}$ length $43 \times 30 \times 41 \mathrm{~mm}^{3}$ ) and

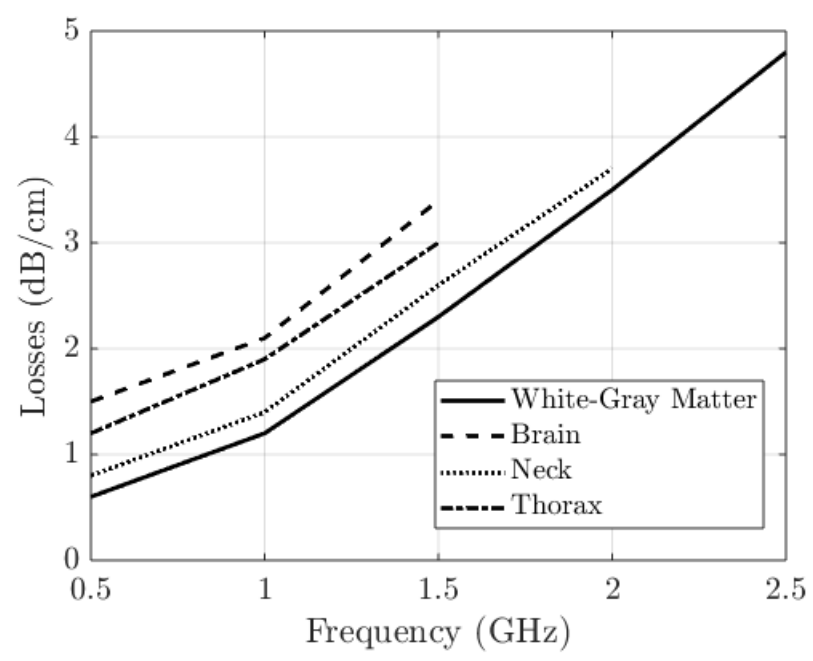

Fig. 8: Measured (EGRH) losses $(\mathrm{dB} / \mathrm{cm})$ of the white-gray matter (Phantom Box), and real brain, neck and thorax of the human volunteer.

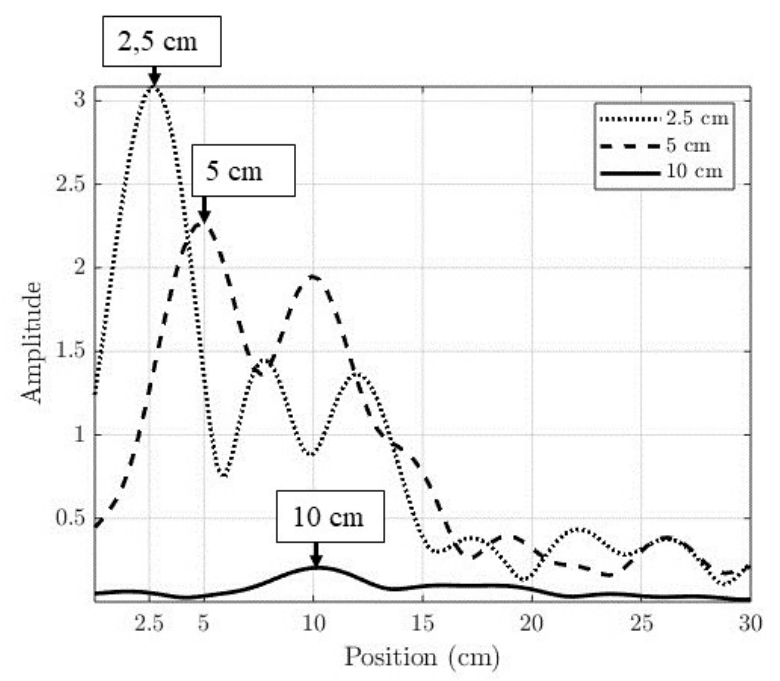

Fig. 9: Time-distance experimental amplitude response extracted from the Reflection measurements showing the capability to detect a metallic object embedded inside of the whitegray matter phantom box at three different positions $(2.5,5$ and $10 \mathrm{~cm})$

unidirectional radiation towards the biological target (above 90\%), which is well suited for a general purpose biomedical antenna probe. The performance of these microwave probes has been experimentally tested by obtaining the propagation losses for different parts of the human body and by detecting an object in an artificial head phantom for depths up to 10 $\mathrm{cm}$. The bodyscope probe may be used into preclinical trials to show real assessment for human or animal bio-medical microwave tests. Future work may involve using the developed microwave probes in a multi-probe array format for microwave imaging. 


\section{REFERENCES}

[1] P. Pedersen, C. Johnson, C. Durney, and D. Bragg, "Microwave reflection and transmission measurements for pulmonary diagnosis and monitoring," IEEE Transactions on Biomedical Engineering, no. 1, pp. 40-48, 1978.

[2] H. Bahrami, E. Porter, A. Santorelli, B. Gosselin, M. Popovich, and L. Rusch, "Flexible sixteen antenna array for microwave breast cancer detection," the breast, vol. 5, no. 8, p. 9, 2015.

[3] M. Guardiola, S. Capdevila, J. Romeu, and L. Jofre, "3-D microwave magnitude combined tomography for breast cancer detection using realistic breast models," IEEE antennas and wireless propagation letters, vol. 11, pp. 1622-1625, 2012.

[4] A. T. Mobashsher, A. M. Abbosh, and Y. Wang, "Microwave system to detect traumatic brain injuries using compact unidirectional antenna and wideband transceiver with verification on realistic head phantom," IEEE Transactions on Microwave Theory and Techniques, vol. 62, no. 9, pp. 1826-1836, 2014.

[5] M. Persson et al., "Microwave-based stroke diagnosis making global prehospital thrombolytic treatment possible," IEEE Transactions on Biomedical Engineering, vol. 61, no. 11, pp. 2806-2817, 2014.

[6] X. Li, M. Jalilvand, Y. L. Sit, and T. Zwick, "A compact double-layer onbody matched bowtie antenna for medical diagnosis," IEEE Transactions on Antennas and Propagation, vol. 62, no. 4, pp. 1808-1816, 2014.

[7] L. Jofre, M. S. Hawley, A. Broquetas, E. de Los Reyes, M. Ferrando, and A. R. Elias-Fuste, "Medical imaging with a microwave tomographic scanner," IEEE Transactions on Biomedical Engineering, vol. 37, no. 3, pp. 303-312, 1990.

[8] S. I. Latif, D. Flores-Tapia, S. Pistorius, and L. Shafai, "Design and performance analysis of the miniaturised water-filled double-ridged horn antenna for active microwave imaging applications," IET Microwaves, Antennas \& Propagation, vol. 9, no. 11, pp. 1173-1178, 2015.

[9] S. I. Latif, D. Flores Tapia, D. Rodriguez Herrera, M. Solis Nepote, S. Pistorius, and L. Shafai, "A directional antenna in a matching liquid for microwave radar imaging," International Journal of Antennas and Propagation, vol. 2015, 2015.

[10] J. Bourqui and E. C. Fear, "Shielded UWB sensor for biomedical applications," IEEE Antennas and Wireless Propagation Letters, vol. 11, pp. 1614-1617, 2012.

[11] U. Schwarz, R. Stephan, and M. A. Hein, "Miniature double-ridged horn antennas composed of solid high-permittivity sintered ceramics for biomedical ultra-wideband radar applications," in Antennas and Propagation Society International Symposium (APSURSI), 2010 IEEE, 2010, pp. 1-4: IEEE.

[12] R. K. Amineh, M. Ravan, A. Trehan, and N. K. Nikolova, "Near-field microwave imaging based on aperture raster scanning with TEM horn antennas," IEEE Transactions on Antennas and Propagation, vol. 59, no. 3, pp. 928-940, 2011.

[13] N. Yatani, K. N. Truong, "BodyScope: A wearable Acoustic Sensor for Activity Recognition.", ACM Internation Conference on Ubiquitous Computing, UbiComp'12, 5-8 Sept., 2012, Pittsburg, USA.

[14] N. Celik, R. Gagarin, G. C. Huang, M. F. Iskander and B. W. Berg, "Microwave stethoscope: development and benchmarking of a vital signs sensor using computer-controlled phantoms and human studies", IEEE Transactions on Biomedical Engineering, vol. 61, no. 8, pp. 2341-2349, 2014.

[15] R. R. Perron, M. F. Iskander, T. B. Seto, G. C. Huang and D. A. Bibb, "Electromagnetics in Medical Applications: The Cardiopulmonary Stethoscope Journey", In The World of Applied Electromagnetics, Springer, pp. 443-479, 2018.

[16] M. Beada'a, A. M. Abbosh, S. Mustafa, and D. Ireland, "Microwave system for head imaging.", IEEE Transactions on Instrumentation and Measurement, vol. 63, no. 1, p. 117, 2014.

[17] D. V. Blackham, R. D. Pollard, An Improved Technique for Permittivity Measurements Using a Coaxial Probe, IEEE Trans. on Instr. Meas., vol. 46, No 5, Oct. 1997, pp. 1093- 1099.

[18] M. Beada'a and A. M. Abbosh, "Realistic head phantom to test microwave systems for brain imaging.", Microwave and Optical Technology Letters, vol. 56, no. 4, pp. 979-982, 2014.

[19] J. Romeu, S. Blanch, N. Vidal, J. M. Lopez-Villegas and A. Agusca, "Assessment of 3D Printing Technologies for Millimeter Wave Reflectors", IEEE Antennas and Wireless Propagation Letters, 2018.

[20] Federal Communication Commission, Body tissue dielectric parameters tool (online), available at http://www.fcc.gov/oet/rfsafety/dielectric/html 